\title{
UNA NUEVA ESPECIE DE BURKSIELLA DE MÉXICO (HYMENOPTERA: TRICHOGRAMMATIDAE)
}

\author{
Verónica Ávila-Rodríguez, ${ }^{1}$ Svetlana N. MYartseva ${ }^{2}$ y Alejandro \\ GONZÁLEZ-HERNÁNDEZ ${ }^{3}$ \\ ${ }^{1}$ Laboratorio de Biología de Invertebrados, Escuela Superior de Biología, Universidad Juárez del \\ Estado de Durango, Ap. Postal 146, Gómez Palacio, Durango, México. \\ ${ }^{2}$ División de Estudios de Postgrado e Investigación, UAM Agronomía y Ciencias, Universidad \\ Autónoma de Tamaulipas, Ap. Postal 87149, Ciudad Victoria, Tamaulipas, México. \\ ${ }^{3}$ Facultad de Ciencias Biológicas, Universidad Autónoma de Nuevo León, Av. Universidad y Pedro de \\ Alba s/n, Ciudad Universitaria, San Nicolás de los Garza, Nuevo León, México. \\ e-mail: vavilar@gmail.com,smyartse@uat.edu.mx, agonzale@fcb.uanl.mx
}

Ávila-Rodríguez, V., S. N. Myartseva \& A. González-Hernández. 2011. Una nueva especie de Burksiella de México (Hymenoptera: Trichogrammatidae). Acta Zool. Mex. (n. s.), 27(1): 77-85. RESUMEN. Se describe una nueva especie de tricogramátido, Burksiella mexicana sp. nov., con base en especímenes colectados en Tamaulipas, México, mediante caracteres morfológicos.

Palabras clave: Burksiella mexicana, nueva especie, Trichogrammatidae, México.

Ávila-Rodríguez, V., S. N. Myartseva \& A. González-Hernández. 2011. A new species of Burksiella from Mexico (Hymenoptera: Trichogrammatidae). Acta Zool. Mex. (n. s.), 27(1): 77-85.

ABSTRACT. A new species of trichogrammatid, Burksiella mexicana sp. nov., is described based on specimens collected in Tamaulipas, Mexico, using morphological characters.

Key words: Burksiella mexicana, new species, Trichogrammatidae, México.

\section{INTRODUCCIÓN}

Burksiella De Santis, 1957, es uno de los géneros poco conocidos de Trichogrammatidae. Se encuentra presente en Asia y en América. En América Burksiella se encuentra en Argentina, Belice, Bolivia, Brasil, Colombia, Costa Rica, Ecuador, Guatemala, México, Nicaragua, Perú, Estados Unidos y Venezuela (Pinto 2006). En México Burksiella está presente en 25 de los 32 estados del país (Pinto 2006, Triapitsyn \& Bernal 2009).

Se han descrito 11 especies de este género, de las cuales, Burksiella chrysomeliphila (Lin 1994) y B. singularis (Yousuf \& Shafee 1988) han sido citadas para Asia y nueve han sido encontradas en el nuevo mundo: $B$. altagraciae Velásquez \& Vig-

Recibido: 13/04/2010; aceptado: 23/08/2010. 
giani (Venezuela), B. benefica (Dozier) (Haití), B. dianae Pinto (México y E.U.A.), B. floridae (Viggiani) (E.U.A), B. ormenidis (Dozier) (Haití), B. platensis (De Santis) (Argentina), B. platysetosa Viggiani \& Velásquez (Venezuela), B. spirita (Girault) (E.U.A.) y B. subannulata De Santis (Argentina) (Pinto 2006).

Las avispitas Burksiella parasitan huevecillos de distintas familias de Hemiptera y Orthoptera. Burksiella ha sido encontrada en huevecillos de hemípteros Fulgoridae sobre sus plantas hospederas Bunchosia, Malphigia y Stigmatophyllum (Malphihiaceae) y de Cicadellidae parasitando a Oncometopia (Similitopia) sp. y Homalodisca liturata Ball, en la planta hospedera Conyza canadensis (L.). También se ha encontrado parasitando huevecillos de ortópteros Tettigoniidae (Pinto 2006). Además, Burksiella ha sido colectada en plantas de jazmín (Dozier 1932, Viggiani 1985, Pinto 2006, Triapitsyn \& Bernal 2009).

El objetivo del presente estudio es describir una nueva especie de Burksiella, que parasita huevecillos de Tettigoniidae en Tamaulipas, México. Esta especie ha sido determinada usando caracteres morfológicos.

\section{MATERIALES Y MÉTODOS}

Colecta de especímenes. Se colectaron huevecillos de Tettigoniidae en el Río San Marcos, Cd. Victoria, Tamaulipas, el 7 de octubre de 2007. Un total de 165 avispitas emergieron.

Procesamiento de especímenes. Las masas de huevecillos fueron colocadas en cámaras de emergencia que consistían en cajas Petri, las muestras de huevecillos fueron revisadas diariamente para la obtención de avispitas, las cuales fueron conservadas en frascos de $20 \mathrm{ml}$ con alcohol al $70 \%$ para su posterior determinación. Para la separación de avispitas tricogramátidas se utilizó un estereoscopio Leica MZ1®.

Determinación morfológica. Algunos de los especímenes obtenidos fueron montados en laminillas con Bálsamo de Canadá de acuerdo a la técnica de montaje en laminilla de Noyes (1982) para calcidoideos (con algunas modificaciones de Svetlana Nikolaevna Myartseva, com. pers.). La observación de los ejemplares se realizó en un miscroscopio Carl Zeizz ${ }^{\circledR}$ modelo Axiostar Plus y objetivo 40x y las microfotografías fueron obtenidas utilizando una cámara Nikon ${ }^{\circledR}$ D100. Las mediciones relativas de la longitud de las estructuras se realizaron aumentando la escala de las imágenes 1000x; es decir, de menos de $0.1 \mathrm{~mm}$ hasta $10 \mathrm{~cm}$. Ejemplares hembras y machos fueron identificados como Burksiella y fueron determinados como especie nueva. Para la determinación a nivel género y especie se revisaron las descripciones originales de Girault (1918), Dozier (1932), De Santis (1957), Viggiani (1985), Yousuf \& Shafee (1988), Lin (1994), Triapitsyn (2003), Pinto (2006), Viggiani \& Velásquez (2007). Especímenes fueron depositados en el Museo de Investigación de Entomología del Departamento de Entomología de la Universidad de California, Riverside, USA (CIUR); Museo de Insectos de la UAM Agronomía y Ciencias, Universidad 
Autónoma de Tamaulipas, Cd. Victoria, Tamaulipas (CIUT) y Colección de Insectos Benéficos Entomófagos, Facultad de Ciencias Biológicas, Universidad Autónoma de Nuevo León, Nuevo León, México (CIBE).

\section{RESULTADOS}

\section{Burksiella mexicana Ávila y Myartseva sp. nov.}

Descripción general. Cuerpo robusto, no relativamente grande, alrededor de $0.5 \mathrm{~mm}$ de longitud. La hembra difiere del macho principalmente en color, la hembra presenta coloración café obscura y el macho coloración amarillo tenue. Ambos presentan dimorfismo sexual antenal muy marcado. La cabeza es ancha, $0.1 \mathrm{x}$ menor que lo ancho del tórax, el macho presenta cabeza megacefálica en relación a la hembra. Antena con dos anellus, dos funículos y una clava de tres segmentos. En la pata las uniones de la coxa, trocánter, fémur y tibia, así como los tres segmentos tarsales, presentan coloración amarillo tenue; la tibia y tarsos moderadamente setosos; fémur menos setoso y reticulado; la metatibia presenta una espuela relativamente delgada, recta y sin bifurcación.

Descripción de la hembra. Coloración café oscuro, excepto la cabeza, antena, escutelo, axila, el metanotum y propodeo, estas estructuras presentan coloración café tenue. Prosoma. La longitud y anchura de la antena son de la siguiente forma: escapo $45 / 17$, pedicelo $23 / 19$, funículo $23 / 37$, clava $74 / 66$. Pedicelo corto, subigual en longitud al primer segmento funicular (F1), con marcaciones de forma estriada, con tres setas largas, presenta dos discos anelli estrechamente unidos a F1. F1 visible sólo en la parte media con tres setas y dos sensilas basicónicas (SB). Segundo segmento funicular (F2) subirregular en forma, agrandado, con sensilas placoideas (SP) grandes, $1.2 x$ más largas que F2, muy visibles y relativamente amplias, se observan por lo menos ocho SP y ocho SB colocadas en forma espaciada no uniforme. La clava presenta tres segmentos de forma subconoidal. El primer segmento de la clava (C1) con $10 \mathrm{SP}$ longitudinales y su parte superior tiene SB colocadas entre las SP; las SP son $1.2 \mathrm{x}$ más largas que $\mathrm{C} 1$; las $\mathrm{SB}$ en forma de hongo; $\mathrm{C} 1$ con setas tan largas como las $\mathrm{SP}$. El segundo segmento de la clava (C2) con al menos seis SP y seis SB en su parte superior, colocadas entre las SP. El tercer segmento de la clava (C3) con seis SP, 1.2x más largas que $\mathrm{C} 3$, con tres SB y con espina terminal (Fig. 1). Mandíbula con tres dientes visibles, palpos maxilares prolongados, con un segmento palpal el cual presenta un par de setas cerca del ápice y una sensila en forma de seta relativamente grande en el ápice. Mesosoma. Lóbulo medio del mesoscuto 1.3x más largo que ancho; lóbulo medio y escutelo con dos pares de setas relativamente largas; lóbulo medio esculpido en forma reticulada; el escutelo unido por una línea transversal; propodeo estrecho con tres setas y marcaciones estriadas; el área del disco subigual en longitud y forma 
que el metanotum; el propodeo presenta un espiráculo con una aparente sensila o prolongación externa longitudinal, de forma cilíndrica. Ala anterior amplia, 1.3x tan larga como ancha, venación $0.52 \mathrm{x}$ de la longitud del ala anterior; setas marginales pequeñas 0.06x de lo ancho del ala anterior (Fig. 2); vena marginal elongada (VM), no extendida apicalmente, con cuatro setas largas en la parte anterior y cinco setas en posición media y posterior; vena premarginal (VPM)con dos setas grandes y sin presencia de sensilas, de forma rectangular, subigual en longitud y amplitud a la VM, presenta un proceso radial marcado, una celda costal con nueve setas en el margen anterior y tres setas en el margen posterior; la VPM y VM separadas por una línea definida y con un espacio blanco entre la separación de ambas, la posición de estas venas está dirigida hacia el margen anterior del ala; vena submarginal (VSM) con una seta relativamente larga; vena estigmal (VE) basalmente estrecha; estigma grande subcuadrangular, $2 \mathrm{x}$ más grande que la $\mathrm{VE}$, presenta un uncus en la parte posterior dirigido hacia la base anterior del ala, con dos sensilas circulares, cada una de las sensilas en apariencia formando dos círculos (Fig. 3); el ala tiene un disco moderadamente setoso; la mayoría de las setas asociadas con las setas lineales (17); Rs1(sector radial 1) presente, con alrededor de 10 setas, elongado, surgiendo del ápice del estigma hacia la base del ala, convergiendo con las venas cubitales (Fig. 2). El ala posterior presenta una estructura simple, disco setoso presente, relativamente amplio, con tres líneas de setas completas, anterior y media (19) y posterior (17) (Fig. 4). Espuela de la metatibia tan larga como el primer segmento tarsal (Fig. 5). Metasoma. Ovipositor: relativamente largo ocupando $3 / 4$ de la porción del abdomen (Fig. 6).

Descripción del macho. Coloración amarillo tenue, excepto el escutum, escutelo, axila, tégula, panel lateral del metanotum y frenum, los cuales son de color café tenue. El mesoscutum presenta dos líneas longitudinales de coloración café, ocupando por lo menos $3 / 4$ partes de la parte superior del mesoscutum. El escutelo presenta estas mismas líneas pero en coloración un poco difusa, no tan marcadas como en el mesoscutum. Prosoma. Cabeza: el macho es megacefálico. La longitud y anchura de la antena son de la siguiente forma: escapo 50/10, pedicelo 23/10, funículo 16/13, clava 37/31. El pedicelo es relativamente largo en comparación con el funículo, con cuatro setas grandes y pronunciadas colocadas en forma longitudinal, con dos anelli, el segundo muy unido a $F 1$. F1 visible completamente, $1.8 x$ más pequeño que $F 2$, sin SP. F2 con seis a ocho SP y al menos seis SB colocadas en la base anterior del funículo en forma intercalada con las SP, que son subiguales en tamaño con respecto a la longitud del funículo. $\mathrm{C} 1$ con seis a ocho SP, con una seta grande en la base anterior. C2 con seis SP, éstas ubicadas no más allá del margen posterior. C3 con cuatro SP. En general, el escapo, pedicelo, funículo y clava presentan más de 10 setas en cada uno de los segmentos, estas setas son moderadamente largas (Fig. 7 y 8). Mesosoma. El macho presenta similitud con la hembra en las distintas estructuras del mesosoma, incluyendo alas (Fig. 9-11). Presenta una espuela en la metatibia que es 3x más corta 

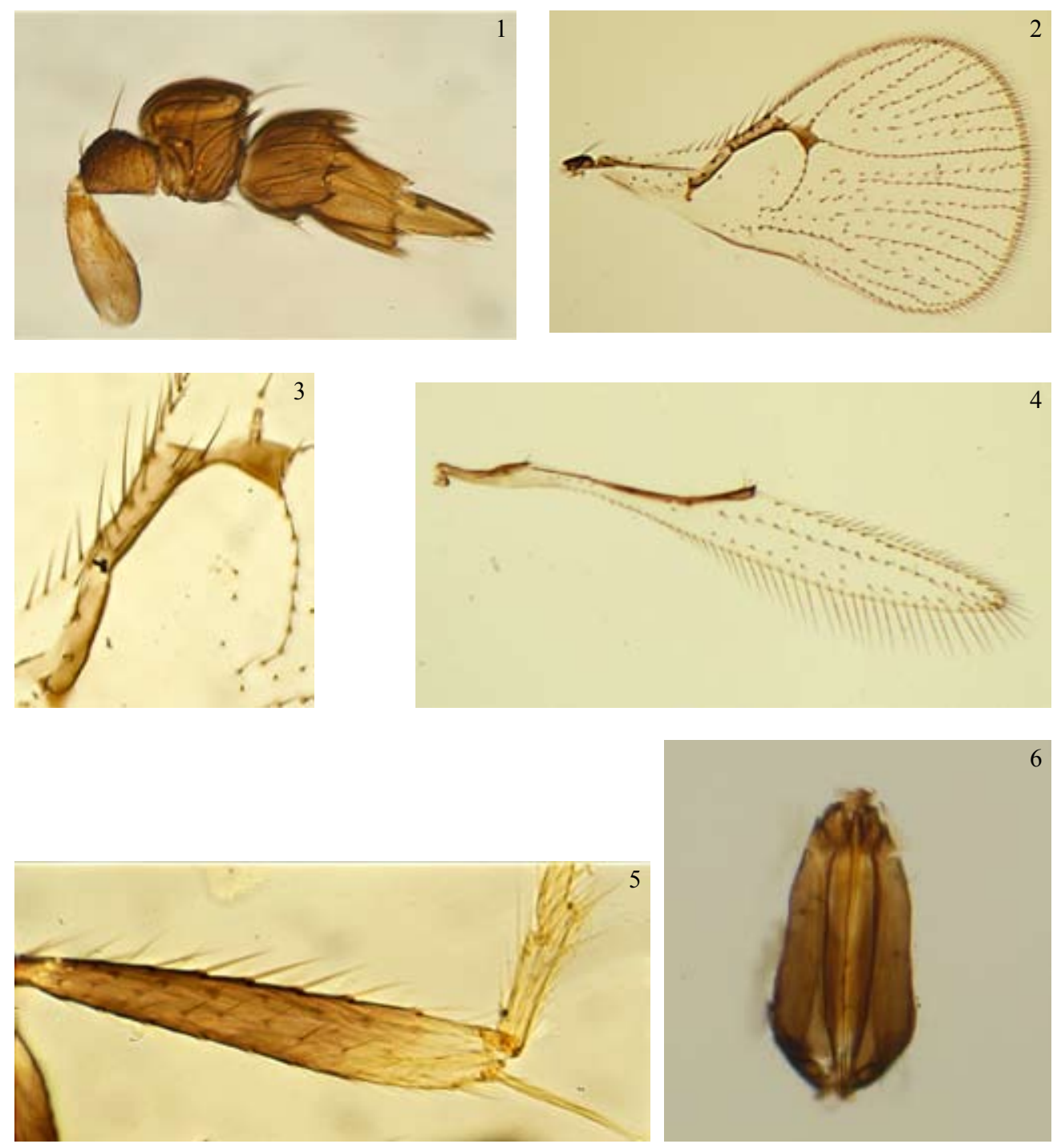

Figuras 1-6. Burksiella mexicana sp. nov., hembra (holotipo): 1) antena, 2) ala anterior, 3) vena del ala anterior, 4) ala posterior, 5) tibia posterior y 6) ovipositor.

que el primer segmento tarsal. Metasoma. Abdomen comprimido, no alargado, con una franja amplia horizontal de color café oscuro, tan amplio como los tres primeros tergitos del gaster, menos robusto que la hembra. Genitalia: Cápsula de la genitalia corta, longitud de la genitalia más corta que la longitud de la tibia anterior, base 

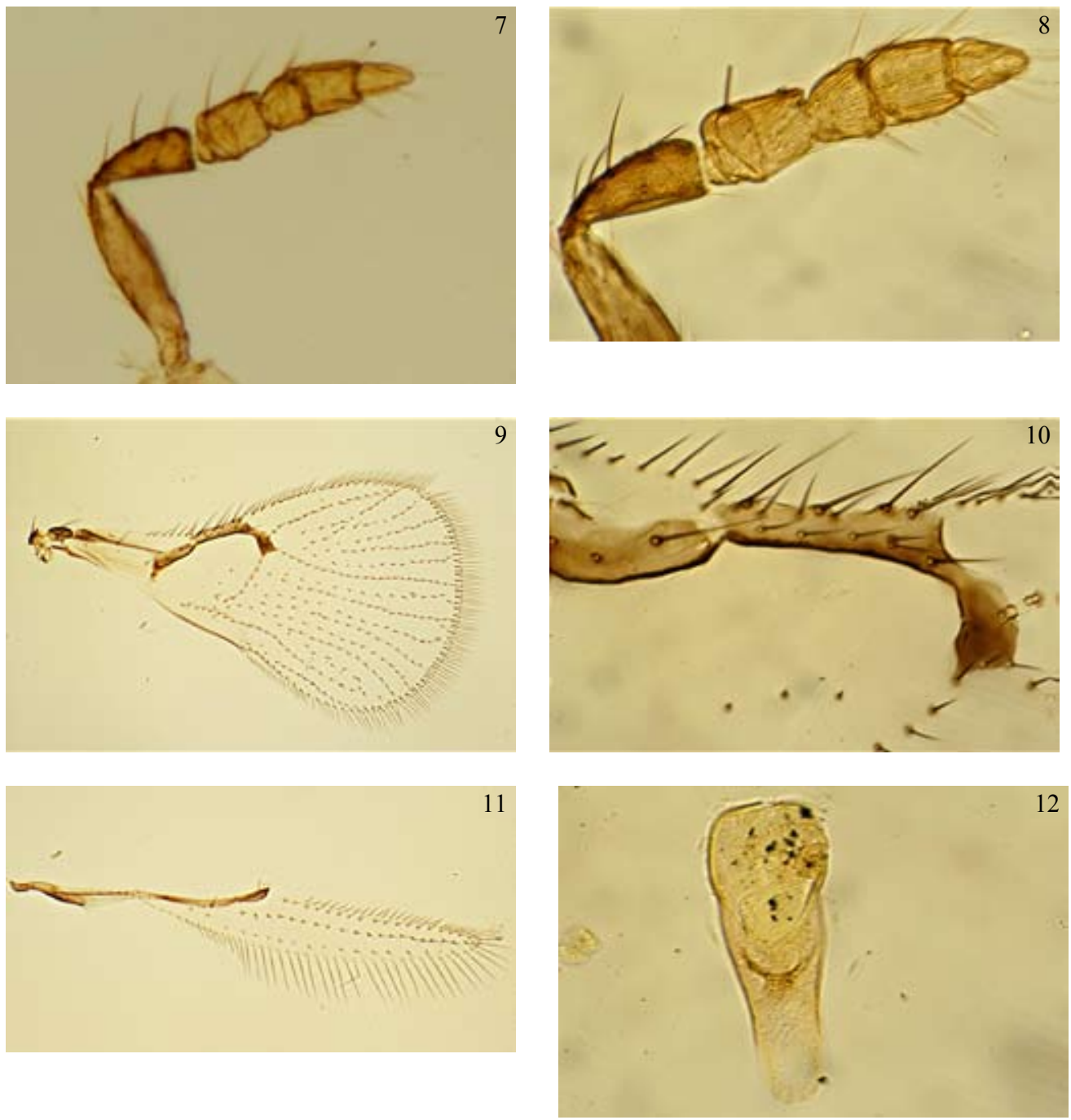

Figuras 7-12. Burksiella mexicana sp. nov., macho: 7 y 8) antena, 9) ala anterior, 10) vena del ala anterior, 11) ala posterior y 12) genitalia.

anterior más amplia que la base posterior, sin presencia de edeago visible, genitalia fusionada en forma de tubo, con un par de lóbulos vestigiales, más dirigidos hacia la base posterior de la genitalia (Fig. 12).

Material tipo: Holotipo hembra, en laminilla. Río de San Marcos, Cd. Victoria Tamaulipas, México. 7/10/2007. Colectado por S. N. Myartseva, de huevecillos de Tettigoniidae; paratipos y alotipo mismos datos de holotipo y (dos machos y una 
hembra en laminilla). El holotipo y un paratipo serán depositados en CIBE, un paratipo en CIUT y el alotipo en CIUR.

Diagnosis. Cuerpo robusto, no extenso, relativamente grande, alrededor de 0.5 $\mathrm{mm}$ de longitud. La hembra difiere del macho principalmente en color, la hembra presenta coloración café obscura y el macho coloración amarillo tenue. Ambos presentan dimorfismo sexual antenal muy marcado. En la antena F2 es subglobular, posee ocho SP de arreglo ligeramente transversal y ocho SB. Las alas anteriores no presentan un área ahumada, el estigma es subcuadrado y la VM posee tres hileras de setas normales, en posición 4, 5, 5 (anterior, medio y posterior, respectivamente). La genitalia del macho no presenta estructuras visibles.

\section{DISCUSIÓN}

Burksiella mexicana sp. nov., puede ser separada de otras especies de Burksiella por que presenta dimorfismo sexual marcado. La hembra posee coloración café oscuro y el macho es amarillo tenue y megacefálico. Estas características sólo las comparte con $B$. benefica una especie encontrada en Haití. La nueva especie $B$. mexicana puede ser distinguida de $B$. benefica porque en las hembras la cabeza, antena, escutelo, axila, metanotum y propodeo son café tenue y el ancho de la cabeza es ligeramente menor $(0.1 \mathrm{x})$ que el ancho del tórax; en los machos el escapo es $5 \mathrm{x}$ más largo que ancho y no posee pigmentación en la base de F1. En B. benefica, en las hembras los márgenes del preescutum, escutelo y márgenes posteriores de las axilas son naranjaamarillento y la cabeza es tan ancha como el tórax; en los machos, el escapo es $2 \mathrm{x}$ más largo que ancho y posee un pigmento rojizo en la base de F1.

Con relación a las especies de Norteamérica, B. mexicana puede ser distinguida de $B$. dianae porque presenta antena con ocho SP en F2, forma del estigma subcuadrada, espacio muy pequeño entre la VM y VPM, éstas de largo y ancho subigual, VM con setas en posiciones 4, 5, 5 (anterior, medio y posterior, respectivamente) y genitalia del macho sin flagelo cuticular; mientras que $B$. dianae presenta cuatro SP en F2, la forma del estigma es suboval, entre la VM y VPM existe un espacio grande, la VM es 1.4x más larga que VPM, la VM presenta setas en las posiciones 3, 4, 4 (anterior, medio y posterior, respectivamente), el macho de esta especie presenta en el margen basal medio de la genitalia un flagelo cuticular corto que se proyecta anteriormente.

Burksiella mexicana se distingue de B. spirita por presentar dimorfismo sexual marcado en la forma de las antenas, la hembra presenta antenas con F2 de forma subglobular, ala anterior 1.3x tan larga como ancha, VM con tres hileras de setas, sin área ahumada detrás de la VM y la VE, y genitalia del macho sin estructuras visibles. En B. spirita no existe dimorfismo sexual marcado en las antenas, en las hembras F2 posee forma subcuadrada, ala anterior $2 \mathrm{x}$ tan larga como ancha, VM con dos hileras de setas, con área ahumada detrás de VM y VE, y genitalia del macho con edeago muy delgado. 
La especie B. mexicana es separada de $B$. floridae por su tamaño de $0.50 \mathrm{~mm}$, VE no elongada distintivamente y genitalia del macho sin estructuras visibles; mientras que $B$. floridae mide alrededor de 0.80 de longitud, VE con una elongación distintiva a manera de cuello, y genitalia con estructuras visibles, ya que posee una falobase medianamente fusionada con el edeago y parámeros en forma de estilete.

Con respecto a las especies de Sudamérica, $B$. mexicana se separa de $B$. altagracia por su tamaño de $0.50 \mathrm{~mm}, \mathrm{~F} 2$ con SP de forma ligeramente transversa, ala posterior con tres líneas de setas y genitalia simple en forma de tubo y sin estructuras visibles. En $B$. altagracia no se presenta dimorfismo sexual marcado, su tamaño promedio es de $0.94 \mathrm{~mm}, \mathrm{~F} 2$ con SP orientadas longitudinalmente y paralelas entre si, ala posterior con cuatro líneas de setas (L1 y L4 incompletas), y genitalia del macho con las siguientes estructuras visiles: falobase corta, parámeros, digitus, edeago corto y apodemas distintivos.

La nueva especie $B$. mexicana se separa de $B$. platisetosa, por: tamaño de 0.50 $\mathrm{mm}, \mathrm{VM}$ del ala anterior con nueve setas normales, F2 de la antena con ocho SP y ocho SB, C1 con 10 SP longitudinales y $10 \mathrm{SB}, \mathrm{C} 2$ con seis $\mathrm{SP}$ y seis SB, C3 con seis $\mathrm{SP}$ y tres SB. En $B$. platisetosa el tamaño promedio es de $0.70 \mathrm{~mm}$, la VM del ala anterior posee un grupo de 12 a 14 setas planas, F2 con dos $\mathrm{SP}$ y tres $\mathrm{SB}, \mathrm{C} 1$ con una $\mathrm{SP}$ y cinco SB, C2 con una SP y una SB y $\mathrm{C} 3$ con tres SP y una SB.

Burksiella subannulata y B. platensis comparten características similares entre si, $B$. mexicana puede ser separada de ellas por presentar las siguientes características: las hembras son de color café oscuro y los machos son de color amarillo tenue, su tamaño es de $0.50 \mathrm{~mm}$, alas anteriores no ahumadas, VE subcuadrada y nervadura marginal normal. En B. subannulata y B. platensis su color es castaño-anaranjado, su tamaño es de $0.60 \mathrm{~mm}$ y $0.71 \mathrm{~mm}$, respectivamente, las alas anteriores ahumadas en casi toda su mitad basal y la VE es de forma redondeada. En particular $B$. subannulata presenta nervaduras de color castaño-amarillento en las alas y la VM es engrosada; mientras que en $B$. platensis las nervaduras son de color oscuro y la VM es angosta.

Burksiella mexicana se diferencia de $B$. ormenidis, por su coloración, la cual es café oscuro en las hembras y amarillo tenue en los machos, ancho de la cabeza ligeramente menor que lo ancho del tórax, antena con $\mathrm{C} 1$ de menor tamaño que el funículo y ala anterior con 17 líneas de setas. En $B$. ormenidis no se observa dimorfismo sexual en coloración, siendo tanto hembras como machos de color amarillo-naranja, la cabeza es tan ancha como el tórax, antena con $\mathrm{C} 1$ más ancha que el funículo y ala anterior con 19 a 20 líneas de setas.

AGRADECIMIENTOS. Se Agradece el apoyo invaluable del doctor Omar G. Alvarado Gómez de la Facultad de Agronomía de la Universidad Autónoma de Nuevo León y al doctor Urbano Nava Camberos de la Facultad de Agricultura y Zootecnia de la Universidad Juárez del Estado de Durango, por las acertadas sugerencias durante la revisión del escrito. Este estudio fue financiado en parte por el Programa 
de Apoyo a la Investigación Científica y Tecnológica (PAICYT) con clave CN359-06 de la Universidad Autónoma de Nuevo León.

\section{LITERATURA CITADA}

De Santis, L. 1957. Descripción de nuevos géneros y especies de calcidoideos Argentinos. II. (Hymenoptera). Notas del Museo de La Plata, Buenos Aires (Zoología), 19: 33-72.

Dozier, H. L. 1932. Descriptions of new trichogrammatid (Hymenoptera) egg parasites from the West Indies. Proceedings of the Entomological Society of Washington, 34: 29-37.

Girault, A. A. 1918. North American Hymenoptera Trichogrammatidae. 11 pp. Privately printed, Sydney, Australia. Reprinted in Gordh, G., A.S. Menke, E.C. Dahms and J.C. Hall. 1979. Memoirs of the American Entomological Institute, 28: 142-152.

Lin, N. Q. 1994. Systematic studies of Chinese Trichogrammatidae. Contributions of the Biological Control. Research Institute, Fujian Agricultural University, Special Publication, 4: 123-239.

Noyes, J. S. 1982. Collecting and preserving chalcid wasps (Hymenoptera: Chalcidoidea). Journal of Natural History, 16: 315-334.

Pinto, J. D. 2006. A review of the New World genera of Trichogrammatidae (Hymenoptera). Journal of Hymenoptera Research, 15: 38-163.

Triapitsyn, S. V. 2003. Taxonomic notes on the genera and species of: (Trichogrammatidae: Hymenoptera)- egg Proconiine sharpshooters (Hemiptera: Clypeorrhyncha: Cicadellidae: Proconiini) in Southeastern U.S.A. Transactions of the American Entomological Society, 129: 245-265.

Triapitsyn, S. V. \& J. S. Bernal. 2009. Egg parasitoids of Proconiini (Hemiptera: Cicadellidae) in Northwestern Mexico, with description of a new species of Gonatocerus (Hymenoptera: Mymaridae). Journal of Insect Science, 9: 1-9.

Viggiani, G. 1985. A new species of Zagella (Hymenoptera: Trichogrammatidae) from Florida. Bollettino del Laboratorio di Entomologia Agraria 'Filippo Silvestri', di Portici, 42: 15-17.

Viggiani, G. \& M. Velasquez. 2007. New Trichogrammatidae (Hymenoptera) from Venezuela. Bollettino del Laboratorio di Entomologia Agraria 'Filippo Silvestri', di Portici, 61: 25-46.

Yousuf, M. \& S. A. Shafee. 1988. Taxonomy of Indian Trichogrammatidae (Hymenoptera: Chalcidoidea). Indian Journal of Systematic Entomology, 4: 55-200. 